\title{
Estimation of an aerosol source in forced ventilation through prior identification of a convolutive model
}

\author{
Preprint - last authors' version - Jan. 8, 2017 \\ International Journal of Heat and Mass Transfer 108 (2017) 1623-1633.
}

\author{
Florent Chata ${ }^{a, b, c}$, Emmanuel Belut ${ }^{c}$, Denis Maillet ${ }^{a, b}$, Francois-Xavier Keller ${ }^{c}$, Anne Taniere ${ }^{a, b}$ \\ a Université de Lorraine, Laboratoire d'Energétique et de Mécanique Théorique et Appliquée (LEMTA) \\ UMR 7563, 2 Avenue de la Forêt de Haye, TSA 60604, Vandoeuvre-lès-Nancy F54518, France \\ b CNRS, Laboratoire d'Energétique et de Mécanique Théorique et Appliquée (LEMTA), UMR 7563, 2 \\ Avenue de la Forêt de Haye, TSA 60604, Vandoeuvre-lès-Nancy F54518, France \\ ${ }^{\mathrm{c}}$ Institut National de Recherche et de Sécurité (INRS), rue du Morvan CS 60027 Vandoeuvre \\ 54519 Cedex, France
}

\section{Abstract}

This article presents a method for estimating the time dependent generation rate of an aerosol source starting from a transient concentration signal measured at a distant point. The method is made up of two distinct steps: a calibration phase, followed by an estimation phase. The calibration phase consists in identifying a transfer function (termed "impedance") between a known source (the "calibration source") and its measured concentration response. In the second step the unknown source generation rate, in the configuration of interest, is estimated by inversion of the corresponding measured concentration signal at the same point, using the previously identified impedance. The time integral of this generation rate, the emitted aerosol dose, can be calculated directly, starting from the integral of the (transient) impedance. Here both simulation of inversions and application to a real experiment have been implemented. The results confirm that it is possible to estimate the temporal pattern of injection and the global emitted mass of pollutant.

Keywords: truncated singular value decomposition, aerosol source, unsteady conditions, inverse problem, computational fluid dynamics, convolutive model 


\section{Introduction}

Wood dust particles are known to be highly carcinogenic [1-2], which makes the study of their emission in the workplace of primary importance for hygiene and safety purposes. According to French data, the number of workers potentially exposed to wood dust lies between 310,000 and 370,000 [3]. Among various sources, hand-held machines produce very high quantities of dust and numerous studies [4-5] have shown that the collection system of most of these machines performs poorly. Assessing the emission rate of these machines is then necessary in order to propose recommendations regarding their choice.

Experimental estimation of the transient intensity of a source through measurement of its response (the concentration) at different locations constitutes an "inverse problem". As in any inverse problem, a direct model has first to be constructed, before considering its inversion. In this study, we consider assessing the particles emission rate during operations such as sanding or sawing of wood in a ventilated workshop.

Modeling wood dust emission by hand-held machines involves many issues:

- the main air flow, created around the machine is turbulent,

- particles are not emitted from a single point source, and the rotation of the sanding tool induces secondary flows,

- particles follow trajectories that may differ from the airflow, depending on their inertia (size, density,...): large particles are deposited near the source while fine particles are dispersed over greater distances,

- aerosol concentration sensors, which provide total concentration measurements or particles size distribution, do not provide really instantaneous responses. Moreover, they must be located away from the source for technical reasons.

Inverse problems can be solved in two distinct ways: the first method is to use a direct detailed modeling and the second refers to the use of a reduced model.

Direct detailed modeling is used here to grasp the main features of the two-phase flow (airflow and particles). However, this model may present some drawbacks for two reasons: the modeled 
concentration is biased for the reasons exposed above and the computing time required is very large, especially when an iterative algorithm is used to solve the inverse problem.

To compensate for the inconveniences of the direct detailed modeling, the construction of a reduced model can be appropriate. There are two ways of constructing a reduced model on a physical basis, here turbulent mass advection and diffusion:

- model reduction, using the knowledge of the detailed model input and output, once a pertinent structure of this reduced model has been selected. This means that the structural parameters of this last model have to be estimated, using generally a non-linear least square procedure,

- model identification, using the measurement of both input and output, in order to estimate the structural parameters of the reduced model. This corresponds to a calibration of the input/output relationship on an experimental basis, the physical system itself being considered as the instrumentation.

Let us note that the previous definitions, where the structure of the "reduced model" is not necessary connected to the structure of a detailed model, that can be coined a "reference model" is a generalized one. A more restricted definition consists in applying mathematical transformations to a detailed model in order to obtain a reduced one, for instance a truncation or selection of dominant modes in a modal or a balanced state space model.

The aim of a reduced model is to reproduce the outputs of the detailed model with a lower number of scalar equations to solve, in order to get a smaller computing time while keeping a good accuracy. They are very useful to solve inverse problems, for example in heat or mass transfer, such as process control, source estimation ...One application of such reduced models consists in estimating the unknown source by inverting the previously identified model. Under some restrictive conditions (steady-state flow, for example, see section 2), the input/output relationship becomes a convolution product, with no need to iterate during either the calibration step or during the source reconstruction step. 
The interested reader can find an overview of these methods in Besselink et al. [6]. Source reconstruction for pollutant convection can be found in groundwater flows, see Atmadja and Bagtzoglou [7] for example or, under atmospheric conditions, see Bocquet [8, 9], Davoine and Boquet [10] and Lushi and Stockie [11]. El Badia et a. [12] dealt with the problem of estimation of both position and intensity of a point source for a linear advection-dispersion-reaction problem and Maalej et al [13] studied the same type of problem in a 1D ventilation case.

Few experimental inverse transient input problems for pollutant advection and diffusion in a ventilated room can be found in the literature. Girault et al. [14] used model identification followed by input reconstruction using gas tracers in a ventilated room. The reduction technique he used, the Modal Identification Method [15] was based on the construction of a series of reduced models having the same structure as the original detailed state-space model (a linear state equation and an output equation), written for several concentration sensors and several sources. Discrimination of 2 sources using 3 sensors was possible using a regularization based on Beck's Function Specification Method (also called Future Time Steps Method) [16]. However, the model identification phase was non-linear, since time constants had to be estimated for the diagonalized reduced models.

In the present paper we consider the same type of configuration and methodology, but instead of estimating a reduced model of given order for each source in the identification step, we model the input/output relationship using a transfer function that relates a given concentration sensor to the timevarying intensity of the pollutant source through a convolution product. The advantage of this formulation is that the inverse model identification problem is now linear. This technique has already been used in 1D by Maalej [13] but we now apply it on an experimental basis in 3D. Here a regularization by Truncated Singular Value Decomposition (TSVD) [17] is implemented, both for the identification step (transfer function estimation in a calibration experiment) and for the source estimation step (inverse input problem, in the real experimental conditions). This specific regularization technique has a very interesting advantage: even if the estimated (time) function is biased, as in any regularization technique, the relative bias in the time integral remains very small, whatever the TSVD truncation order. This allows to get estimates of the emitted pollutant dose (integrated intensity) which do not suffer from this type of bias. 


\section{Impedances in advection-diffusion problems in mass transfer}

\subsection{Convolutive response}

A very interesting mathematical property of systems of partial differential equation (PDE) with time independent coefficients is the convolutive nature of their solution.

This will be shown here for the generic mass transfer equation of a species (a pollutant in air) whose mass concentration $C$ (in kg.m ${ }^{-3}$ for example) depends both on time $t$ and on the current point $\mathrm{P}$ in space:

$\frac{\partial C}{\partial t}(\mathrm{P}, t)+\vec{u}(\mathrm{P}) \cdot \vec{\nabla}\left(D_{\text {eff }} \vec{\nabla} C(\mathrm{P}, t)\right)+\frac{Q(t)}{V_{\text {source }}} \chi_{\text {source }}(\mathrm{P})$ where $\left\{\begin{array}{l}\chi_{\text {source }}(\mathrm{P})=1 \text { if } \mathrm{P} \in \Omega_{\text {source }} \\ \chi_{\text {source }}(\mathrm{P})=0 \text { elsewhere }\end{array}\right.$

The initial concentration at time $t=0$ is supposed to be equal to zero in the considered domain, a room for example, and the boundary conditions are supposed to be homogeneous: zero concentration at the inlet(s) and zero normal gradient at the outlet(s) and at the walls, for example.

A unique pollutant source emits a mass flow rate $Q(t)$ of pollutant (in $\mathrm{kg}_{\mathrm{s}}{ }^{-1}$ ), called the intensity (or the time dependent generation rate) of the source, over a given subdomain $\Omega_{\text {source }}$ of volume $V_{\text {source }}$ defined by its characteristic function $\chi_{\text {source }}(\mathrm{P})$. The local velocity field $\vec{u}(\mathrm{P})$ is supposed to depend on space but not on time (steady flow). $D_{\text {eff }}(\mathrm{P})$ in equation (1) is the effective mass diffusion coefficient that is the sum of its molecular and turbulent components.

Equation (1) associated with its initial and boundary conditions can be submitted to a Laplace transformation, which gives rise to a PDE system depending on space only, for a fixed value of the Laplace parameter $p$. There is just a single source term in this system, that is proportional to the Laplace transform $\bar{Q}(p)$ of the initial intensity $Q(t)$ of the source (we use here an upper bar to designate the Laplace transform of any time dependent quantity).

As a consequence, the new PDE system being linear, the Laplace transform $\bar{C}(P, p)$ of the local concentration $C(\mathrm{P}, t)$ at any point $\mathrm{P}$, is proportional to $\bar{Q}(p)$, which can be expressed by equation (2a):

$$
\bar{C}(\mathrm{P}, p)=\bar{Z}(\mathrm{P}, p) \bar{Q}(p)
$$




$$
\Rightarrow C(\mathrm{P}, t)=Z(\mathrm{P}, t) * Q(t)=\int_{0}^{t} Z\left(\mathrm{P}, t-t^{\prime}\right) Q\left(t^{\prime}\right) \mathrm{d} t^{\prime}
$$

The proportionality constant $\bar{Z}$ is the Laplace transform of a transfer function $Z$ in the time domain. So, in this domain, equation (2b) shows that the local concentration $C$, that is the forced response to the unique transient input $Q$, is a convolution product (designated by the star character "*) in equation (2b)) between this local transfer function $Z$, called here an impedance, and the intensity $Q$ of the source.

\subsection{Discretized column vector/matrix model and parameterization of source and impedance}

Model (2.b) is continuous in time. In the inverse approach, it has to be associated to $m$ discrete observations at measurement times $t_{i}=t_{0}+i \Delta t$ (for $i=1$ to $m$ ), where $t_{0}$ is the initial time, that corresponds to a zero concentration, and $\Delta t$ the time step. So, the discretization of the local concentration at a specific observed point $\mathrm{P}_{o b s}$ becomes:

$$
C_{i}=C\left(\mathrm{P}_{\text {obs }}, t_{i}\right) \text { for } i=1 \text { to } m
$$

In a similar way, the unknowns of the inverse problem, that is the local impedance for the identification problem and the intensity of the source for the inverse input problem, are parameterized on a finite basis of $m$ piecewise constant functions $f_{j}$ :

$$
Q(t)=\sum_{j=1}^{m} Q_{j} f_{j}(t) \text { and } Z(t)=\sum_{j=1}^{m} Z_{j} f_{j}(t) \text { where } f_{j}(t)=\mathrm{H}\left(t-t_{j-1}\right)-\mathrm{H}\left(t-t_{j}\right) \text { for } j=1 \text { to } m
$$

where $\mathrm{H}$ designates the Heaviside function. This means that both $Q_{j}$ and $Z_{j}$ are then mean values of original functions $Q(t)$ and $Z(t)$ over each $\left[\begin{array}{ll}t_{j-1} & t_{j}\end{array}\right]$ time interval :

$$
Q_{j}=\frac{1}{\Delta t} \int_{t_{j-1}}^{t_{j}} Q(t) \mathrm{d} t \quad \text { and } \quad Z_{j}=\frac{1}{\Delta t} \int_{t_{j-1}}^{t_{j}} Z(t) \mathrm{d} t
$$


If the time step $\Delta t$ is small enough, to be adapted to the time scales of both input $Q(t)$ and impedance $Z(t)$, in order to grasp their possible sharp variations in time, which is important for the inverse input problem, it is possible to approximate equation (2b), written at time $t_{i}$ by a numerical quadrature :

$$
C_{i} \approx \Delta t \sum_{j=1}^{i} Z_{i-j+1} Q_{j}
$$

This scalar model can be put under a column vector/matrix form:

$$
C=\mathbf{M}(\boldsymbol{Q}) \mathbf{Z}
$$

or, alternatively

$$
C=\mathbf{M}(Z) \boldsymbol{Q}
$$

where each column vector $\boldsymbol{C}, \boldsymbol{Q}$ or $\boldsymbol{Z}$ are composed of the $m$ scalars $C_{i}, Q_{i}$ or $Z_{i}$ and where $\mathbf{M}($.$) is a (square) matrix function of a column vector, here a Toeplitz matrix defined for any column$ vector $\boldsymbol{x}$ as :

$$
\mathbf{M}(\boldsymbol{x}) \equiv \Delta t\left[\begin{array}{ccccc}
x_{1} & & & & \\
x_{2} & x_{1} & & 0 & \\
x_{3} & x_{2} & x_{1} & & \\
\vdots & \vdots & \vdots & \ddots & \\
x_{m} & x_{m-1} & x_{m-2} & \cdots & x_{1}
\end{array}\right] \quad \text { where } \quad \boldsymbol{x}=\left[\begin{array}{c}
x_{1} \\
x_{2} \\
x_{3} \\
\vdots \\
x_{m}
\end{array}\right]
$$

Let us note that $Q$ and $Z$ play a symmetrical role in equations (6a and b) because of the commutative property of the convolution product. Equation (6a) will be used as the linear model in the identification of impedance $Z$ (calibration experiment with a known $Q$ ) while equation (6b) will constitute another model for the estimation of source $Q$ (inverse input problem in the experiment one is interested in).

\subsection{Link between transient and steady state models through the notion of dose}

In steady state regime, concentration $C^{s s}$ at any point in the system is proportional to the emission rate $Q_{0}$ : 


$$
C^{s s}=R Q_{0}
$$

where constant $R$ can be considered as a pseudo resistance to mass transfer. One way to achieve this type of regime, starting from a zero initial concentration everywhere in the physical system, is to assume that the emission rate $Q(t)$ is a step function of time, which yields:

$$
Q(t)=Q_{0} H(t) \Rightarrow \bar{Q}(p)=Q_{0} / p
$$

On the one hand, substitution of this Laplace mass flow rate of pollutant into equation (2a) gives, dropping the $\mathrm{P}$ argument:

$$
p \bar{C}(p)=\bar{Z}(p) Q_{0} \Rightarrow \lim _{\text {as } p \rightarrow 0}[p \bar{C}(p)]=\lim _{\text {as } p \rightarrow 0}\left[\bar{Z}(p) Q_{0}\right]=\bar{Z}(0) Q_{0}
$$

On the other hand, the final value theorem (a property of the Laplace transformation) writes out :

$$
\lim _{\text {as }}[p \rightarrow 0 \text { C }(p)]=\lim _{\text {as } t \rightarrow+\infty}[C(t)]=C^{s s}
$$

Hence :

$$
\bar{Z}(0) Q_{0}=C^{s s}
$$

Comparison (7) and (10) yields:

$$
R=\bar{Z}(0)=\int_{0}^{+\infty} \exp (-0 t) Z(t) \mathrm{d} t
$$

We will designate dose, noted $D_{x}$ here, the time integral of any time-varying quantity $x(t)$ :

$$
D_{x}=\int_{0}^{+\infty} x(t) \mathrm{d} t \text { for } x=Z, Q \text { or } C
$$

Let us note that the notion of dose of $x$ here is the same as the notion of moment of zero order. So, equation (11) can be written the following way:

$$
R=D_{Z}
$$

which means that the steady state resistance is simply the dose of the (transient) impedance. Of course integral (12) has to be convergent, which is the case for $D_{Z}$ that is input independent.

However in order for $D_{Q}$ and $D_{C}$ to exist, the input $Q(t)$ has to go back to zero after a finite time. Under these conditions, equation (2a) written for a zero Laplace parameter $p$ can be expressed in terms of doses:

$$
D_{C}=D_{Z} D_{Q}
$$




\section{Regularization of the two deconvolution problems}

\subsection{Ordinary Least Square solutions for the identification problem}

Both identification and estimation problems are inverse deconvolution problems. Their models have the same structure. Let us consider first the identification problem. In the calibration experiment, the measured signal $\boldsymbol{Y}^{\text {cal }}$ is composed of the exact output $\boldsymbol{C}^{\text {exact }}$ augmented by an additive noise $\boldsymbol{\varepsilon}$. In a column vector notation, that yields:

$$
\boldsymbol{Y}^{\text {cal }}=\boldsymbol{C}^{\text {exact }}+\boldsymbol{\varepsilon} \quad \text { where } \quad \boldsymbol{C}^{\text {exact }}=\mathbf{M}\left(\boldsymbol{Q}^{\text {cal }}\right) \boldsymbol{Z}^{\text {exact }} \text { and } \mathrm{E}(\boldsymbol{\varepsilon})=\mathbf{0}
$$

In this equation $\boldsymbol{Q}^{\text {cal }}$ designates the measured input, supposed noiseless here and $E()$ the expectation of a random quantity. So, equation (6a) being square, the Ordinary Least Square (OLS) solution is:

$$
\hat{\boldsymbol{Z}}_{O L S}=\operatorname{Arg}\left(\min \left(J_{O L S}(\boldsymbol{Z})\right)\right)=\boldsymbol{M}^{-1} \boldsymbol{Y}^{\text {cal }} \text { with } J_{O L S}(\boldsymbol{Z})=\left\|\boldsymbol{Y}^{\text {cal }}-\mathbf{M}\left(\boldsymbol{Q}^{\text {cal }}\right) \boldsymbol{Z}\right\|_{2}^{2} \text { and } \boldsymbol{M}=\mathbf{M}\left(\boldsymbol{Q}^{c a l}\right)
$$

where subscript "2" designates the Euclidian norm and matrix $\boldsymbol{M}$ the value of the Toeplitz matrix function $\mathbf{M}\left(\boldsymbol{Q}^{c a l}\right)$ constructed with column-vector $\boldsymbol{Q}^{\text {cal }}$, see equation (6c).

It is easy to see that this identification problem is ill-posed since the determinant of the lower triangular matrix $\mathbf{M}\left(\boldsymbol{Q}^{\text {cal }}\right)$ is equal to $\left(Q_{1}^{\text {cal }} \Delta t\right)^{m}$. So, the choice of the initial time $t_{0}$ is important: it has to be chosen prior to the time when $Q^{\text {cal }}(t)$ departs from zero. However, if its average value in the $\left[t_{0}, t_{1}\right]$ interval is small, the determinant of this matrix becomes close to zero and the deconvolution problem becomes ill-posed ( $\boldsymbol{Y}^{\text {cal }}$ instead of $\boldsymbol{C}^{\text {exact }}$ ), with large standard deviations for the different components of the estimated impedance vector $\hat{\mathbf{z}}_{O L S}$.

The same type of remark can be made for the choice of the time step $\Delta t$ : a low value ensures a good resolution for the parameterization (4) of both functions $Q(t)$ and $Z(t)$ with an unbiased model (2b) for the response $C(t)$. However, concomitantly, such a choice would make the inversion ill-posed in case of noise in this response, so a regularization is necessary. We have chosen to use a Truncated Singular Value Decomposition (TSVD) here. 


\subsection{TSVD regularization of the identification problem}

If the Singular Value Decomposition (SVD) of square matrix $\boldsymbol{M}$ is written, see equation (17a) below, it is easy to show that the OLS estimate of impedance $\boldsymbol{Z}(15)$ is given by equation (17b):

$$
\begin{aligned}
\boldsymbol{M} & =\boldsymbol{U} \boldsymbol{W} \boldsymbol{V}^{t} \\
\Rightarrow \quad \hat{\boldsymbol{Z}}_{\text {OLS }} & =\boldsymbol{V} \boldsymbol{W}^{-1} \boldsymbol{U}^{t} \boldsymbol{Y}^{c a l}
\end{aligned}
$$

In these equations, all three matrices are $(m \times m), \boldsymbol{W}$ being diagonal (with its singular values numbered in decreasing order: $\left.\quad w_{1} \geq w_{2} \geq \cdots \geq w_{m-1} \geq w_{m}\right)$ and $\boldsymbol{U}$ and $\boldsymbol{V}$ being orthogonal. This identity is valid only if matrix $\boldsymbol{M}$ is of full rank, which means that its smaller singular value $w_{m}$ should be strictly positive.

In practice, the Euclidian condition number cond $(\boldsymbol{M})=w_{1} / w_{m}$ is very large, or even infinite for a system where the response time of the system (here the pollutant source/concentration sensor) is small, see section 3.1, and a regularization is always necessary. If Truncated SVD is used, only a number $\alpha$ of singular values has to be kept in a truncated version of equation (17), with $\alpha$ smaller or equal to the rank $r$ of the system, yielding the regularized estimator $\hat{\boldsymbol{z}}_{\alpha}$ :

$$
\begin{gathered}
\hat{\boldsymbol{Z}}_{\alpha}=\boldsymbol{V}_{A} \boldsymbol{W}_{A}^{-1} \boldsymbol{U}_{A}^{t} \boldsymbol{Y}^{\text {cal }} \text { with } \boldsymbol{W}_{A}=\operatorname{diag}\left(\left[\begin{array}{llll}
w_{1} & w_{2} & \cdots & w_{\alpha}
\end{array}\right]\right) \text { and } \alpha<r \leq m \\
\text { where } \boldsymbol{U}_{A}=\left[\begin{array}{lllll}
\boldsymbol{U}_{1} & \boldsymbol{U}_{2} & \cdots & \boldsymbol{U}_{\alpha}
\end{array}\right] \text { and } \boldsymbol{V}_{A}=\left[\begin{array}{llll}
\boldsymbol{V}_{1} & \boldsymbol{V}_{2} & \cdots & \boldsymbol{V}_{\alpha}
\end{array}\right]
\end{gathered}
$$

Here matrices $\boldsymbol{U}_{A}$ and $\boldsymbol{V}_{A}$ are composed of the $\alpha$ first left and right singular column vectors $\boldsymbol{U}_{k}$ and $\boldsymbol{V}_{k}$ respectively, corresponding to the $\alpha$ largest singular values $w_{k}$ (for $k=1$ to $m$ ).

An alternate less classical but equivalent version of this TSVD estimator is:

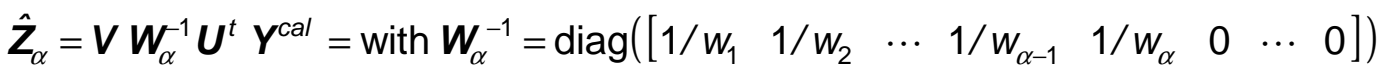

$$
\begin{aligned}
& \text { with } \alpha<r \leq m
\end{aligned}
$$

The interest of this version is to keep the matrices of the left and right singular vectors unchanged in this TSVD regularized estimate: the notation $\boldsymbol{W}_{\alpha}^{-1}$ is only symbolic here since diagonal 
matrix $\boldsymbol{W}_{\alpha}$ does not exist as it corresponds to matrix $\boldsymbol{W}$ in which the last $(m-\alpha)$ (smallest) singular values have been given infinite values.

The choice of the optimal regularization hyperparameter $\alpha$ is made according to Morozov's discrepancy principle [18], in the case of an independent identically distributed (i.i.d.) noise of standard deviation $\sigma$. So, $\alpha$ is chosen so that:

$$
J\left(\hat{\mathbf{Z}}_{\alpha}\right)<m \sigma^{2} \text { and } J\left(\hat{\mathbf{Z}}_{\alpha+1}\right) \geq m \sigma^{2}
$$

\subsection{Estimation for the inverse input problem}

Once a regularized estimation, called $\hat{\boldsymbol{Z}}$ now on, of the impedance vector $\boldsymbol{Z}$ has been obtained through the previous calibration experiment, the convolution model written under its form (6b) is used for estimating the intensity of the source $\boldsymbol{Q}^{\text {real }}$ in the experiment one is interested in, that is called 'real experiment' here. So, in case of a noise $\boldsymbol{\varepsilon}$ in the unbiased concentration signal $\boldsymbol{Y}$, one gets:

$$
\boldsymbol{Y}=\boldsymbol{C}^{\text {exact }}+\boldsymbol{\varepsilon} \quad \text { where } \quad \boldsymbol{C}^{\text {exact }}=\mathbf{M}\left(\boldsymbol{Z}^{\text {exact }}\right) \boldsymbol{Q}^{\text {real }} \text { and } E(\boldsymbol{\varepsilon})=\mathbf{0}
$$

The procedure for estimating $\boldsymbol{Q}^{\text {real }}$ is the same as above, in section 3.2, with the OLS estimator:

$$
\begin{gathered}
\hat{\boldsymbol{Q}}_{O L S}^{\text {real }}=\operatorname{Arg}\left(\min \left(J_{O L S}\left(\boldsymbol{Q}^{\text {real }}\right)\right)\right)=\boldsymbol{M}^{-1} \boldsymbol{Y} \\
\text { with } J_{O L S}\left(\boldsymbol{Q}^{\text {real }}\right)=\left\|\boldsymbol{Y}-\mathbf{M}(\hat{\boldsymbol{Z}}) \boldsymbol{Q}^{\text {real }}\right\|_{2}^{2} \text { and } \boldsymbol{M}=\mathbf{M}(\hat{\boldsymbol{Z}})
\end{gathered}
$$

This can be regularized by TSVD, using the SVD decomposition of $\mathbf{M}(\hat{\boldsymbol{Z}})$ :

$$
\hat{\mathbf{Q}}_{\alpha}^{\text {real }}=\boldsymbol{V} \boldsymbol{W}_{\alpha}^{-1} \boldsymbol{U}^{t} \boldsymbol{Y} \text { with } \mathbf{M}(\hat{\boldsymbol{Z}})=\boldsymbol{U} \boldsymbol{W} \boldsymbol{V}^{t}
$$

\subsection{Estimation of the doses}

There are two ways for estimating the doses of both impedance and input for the two experiments (calibration and real experiments).

The first one, noted $\boldsymbol{a}$ here, relies on the preceding TSVD inversions of the two deconvolution problems:

- $\quad$ for the calibration experiment, using $\hat{\boldsymbol{z}}_{\alpha}$ given by equation (18b):

$$
\hat{D}_{Z}^{a}=\Delta t \boldsymbol{u}_{m} \hat{\boldsymbol{z}}_{\alpha} \quad \text { where } \quad \boldsymbol{u}_{m}=\left[\begin{array}{llll}
1 & 1 & \cdots & 1
\end{array}\right] \text { with } \boldsymbol{u}_{m} \in \mathbb{R}^{m}
$$


- for the real experiment, using $\hat{\mathbf{Q}}_{\alpha}^{\text {real }}$ given by equation (21):

$$
\hat{D}_{Q}^{a}=\Delta t \boldsymbol{u}_{m} \hat{\boldsymbol{Q}}_{\alpha}^{r e a l}
$$

The second one, noted $\boldsymbol{b}$ here, stems directly from equation (14) and is detailed below:

- for the calibration experiment, using direct integration of the doses of the experimental (noisy) concentration $\boldsymbol{Y}^{\text {cal }}$ and on the (exact) measured calibration source $\boldsymbol{Q}^{\text {cal }}$ defined by equation (15):

$$
\hat{D}_{Z}^{b}=D_{Y}^{c a l} / D_{Q}^{c a l} \text { where } D_{Y}^{c a l}=\Delta t \boldsymbol{u}_{m} \boldsymbol{Y}^{\text {cal }} \text { and } D_{Q}^{\text {cal }}=\Delta t \boldsymbol{u}_{m} \boldsymbol{Q}^{c a l}
$$

- for the real experiment, using the experimental (noisy) concentration $Y$ defined by equation (19):

$$
\hat{D}_{Q}^{b}=D_{Y} / \hat{D}_{Z}^{b} \text { where } D_{Y}=\Delta t \boldsymbol{u}_{m} \boldsymbol{Y}
$$

The interest of the $\boldsymbol{b}$ estimates is that they correspond to a scalar well-posed problem, which is not the case for the a estimates that are the by-products of two ill-posed function estimation problems.

\subsection{Summary: Implementation}

To summarize, the field implementation of this method consists of following these steps:

1. Impedance calibration

For this step, the impedance is estimated from a calibrated source $\boldsymbol{Q}^{\text {cal }}$ and implies the measurement $\boldsymbol{Y}^{\text {cal }}$ of concentration at a given point in the flow. Estimation of the impedance is carried out using TSVD regularization, with the truncation order used noted $\alpha_{1}$, which yields:

$$
\hat{\boldsymbol{Z}}_{\alpha_{1}}=\left(\mathbf{M}_{\alpha_{1}}\left(\boldsymbol{Q}^{c a l}\right)\right)^{-1} \boldsymbol{Y}^{c a l}
$$

where $\left(\mathbf{M}_{\alpha_{1}}\left(\boldsymbol{Q}^{\text {cal }}\right)\right)^{-1}$ designates the truncated SVD version (with $\alpha_{1}$ singular values kept) of the inverse of matrix $\mathbf{M}\left(\boldsymbol{Q}^{\text {cal }}\right)$

2. Estimation of unknown generation rate 
In this step corresponding to the experiment with an unknown generation rate, the previously estimated impedance $\hat{Z}_{o_{1}}$ is used with measurement $\boldsymbol{Y}$ of concentration at the same point used for calibration. The second truncation order used is noted $\alpha_{2}$ and the unknown generation rate estimation is calculated such as:

$$
\hat{\mathbf{Q}}_{\alpha_{2}}^{\text {real }}=\left(\mathbf{M}_{\alpha_{2}}\left(\hat{\boldsymbol{Z}}_{\alpha_{1}}\right)\right)^{-1} \boldsymbol{Y}
$$

\section{Experimental configuration}

The present method for aerosol source estimation was applied to the case of an open ventilated cabin where an aerosol source is present (Figure 1).

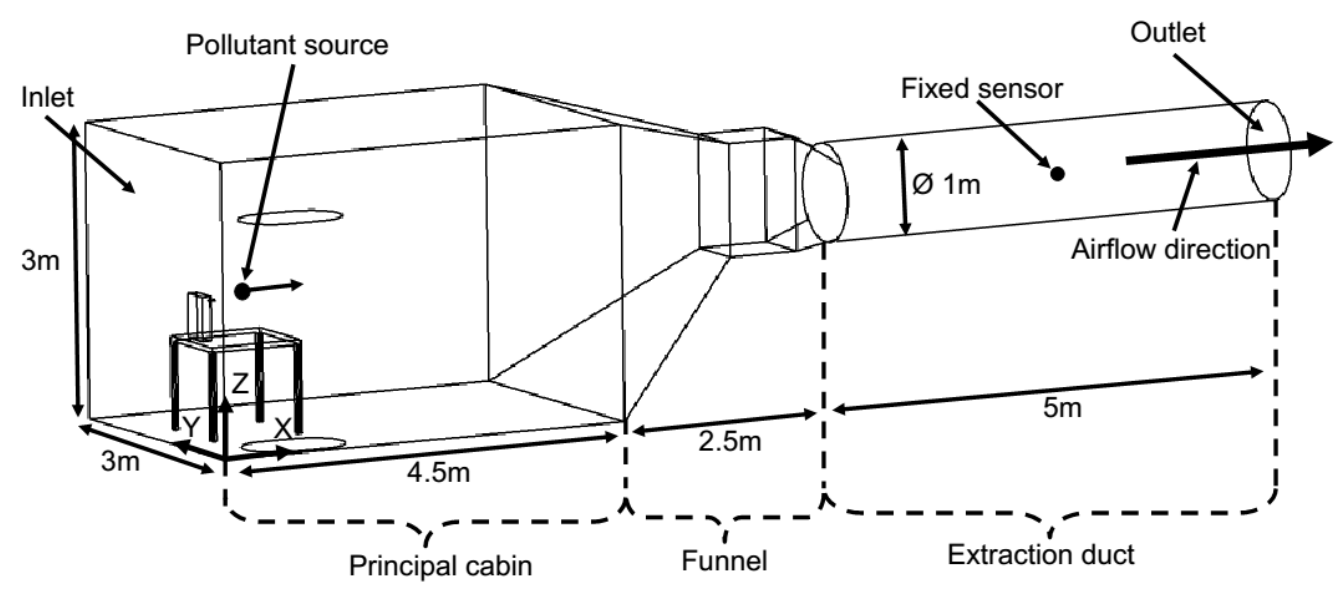

Figure 1 - Diagram of the ventilated open cabin used

The dimensions of the principal cabin are depicted in figure 1. A source of particulate pollutant is placed close to the inlet. This principal cabin is connected to the extraction duct through a funnel-like part. This duct is $5 \mathrm{~m}$ long with a $1 \mathrm{~m}$ diameter. The total length of the open ventilated cabin (principal cabin, funnel, and extraction duct) is $12.5 \mathrm{~m}$. The cabin operated at a flow rate of $9,720 \mathrm{~m}^{3} \cdot \mathrm{h}^{-1}$ (that is a mean velocity of $0.3 \mathrm{~m} \mathrm{~s}^{-1}$ at the inlet section). A vertical diffusing canvas has been set in the inlet section in order to ensure a uniform horizontal velocity distribution.

The calibrated aerosol source is a Palas ${ }^{\circledR}$ RBG-1000 generator to which a small divergent nozzle was added. In this generator, the particles placed in a reservoir are conveyed onto a rotating 
brush at a precisely controlled feed rate and an adjustable secondary volume flow disperses the particles into the main flow through the cabin. Thus, the air charged with airborne particles is ejected horizontally at an average velocity of $0.55 \mathrm{~m} . \mathrm{s}^{-1}$ over a circular cross-section of $40 \mathrm{~mm}$ diameter. The particle injection velocity is quite low in order not to modify the main flow. The aerosol source was placed at a distance of $X=0.9 \mathrm{~m}$ from the inlet of the cabin, at a distance $Y=1.5 \mathrm{~m}$ from the wall (that is at the middle of the cabin in the $Y$ direction) and at a height of $Z=1.4 \mathrm{~m}$. For all cases considered in the present paper, particles used are a recycled sanding wood dust whose size distribution is shown in Figure 2. This dust was characterized using an Aerodynamic Particle Sizer (APS/TSI ${ }^{\circledR}-3321$ ). This sensor provides high-resolution, real-time aerodynamic measurements of the distribution of the particles in the 0.5 to 20 micrometer range (see Figure 2) thanks to an optical measurement based on the acceleration of the particles.

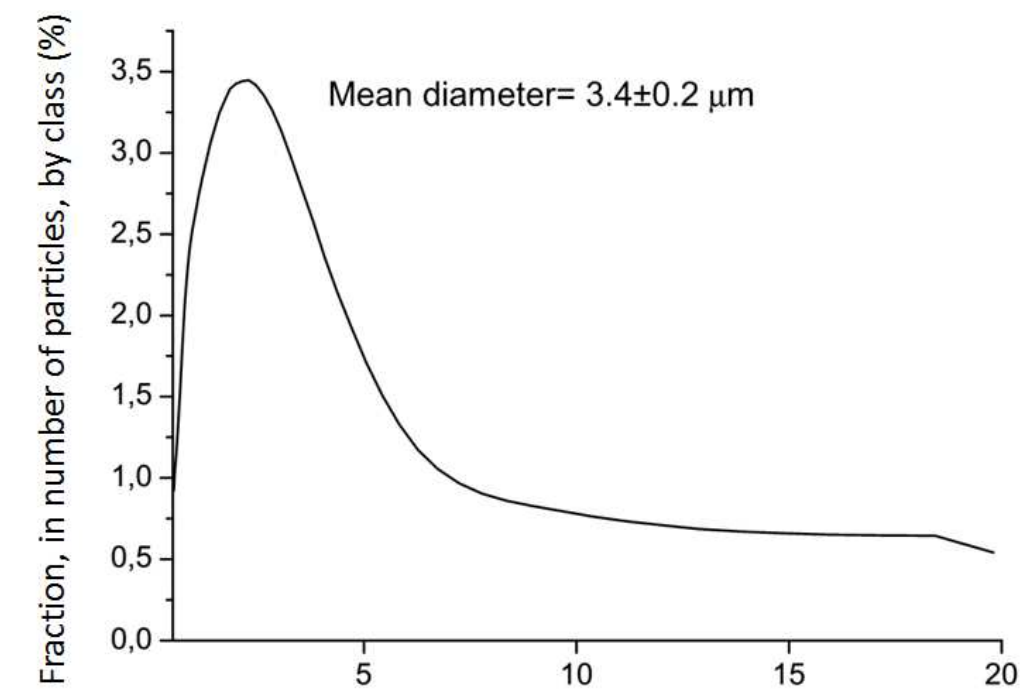

Particle aerodynamic diameter $(\mu \mathrm{m})$ - 52 classes between 0.5 and $20 \mathrm{~mm}$

Figure 2 - Size distribution of sanding wood dust

The position of the measurement point is located in the outlet duct (Fixed sensor at $X=11 \mathrm{~m}$, see Figure 1). This position was chosen because this exhaust duct is the region where the aerosol is best mixed: this makes the corresponding measurement of the transient concentration at this point a good sample of the average particle transport in the cabin, and it also makes this measurement point less sensitive to unsteady perturbations. 
Prior to implementing the method directly on real measurements in an experimental cabin, it was firstly applied to the concentration output of a CFD model of this cabin which provides results without experimental uncertainties (no measurement noise or bias).

\section{Numerical validation using synthetic measurements}

For this CFD modeling application, the studied source corresponds to various generation rates of the source shown in Figure 3. In the numerical simulation, the particles used are monodispersed with a diameter equal to the mean diameter of sanding wood dust ( $\left.d_{p}=3.4 \mu \mathrm{m}\right)$.

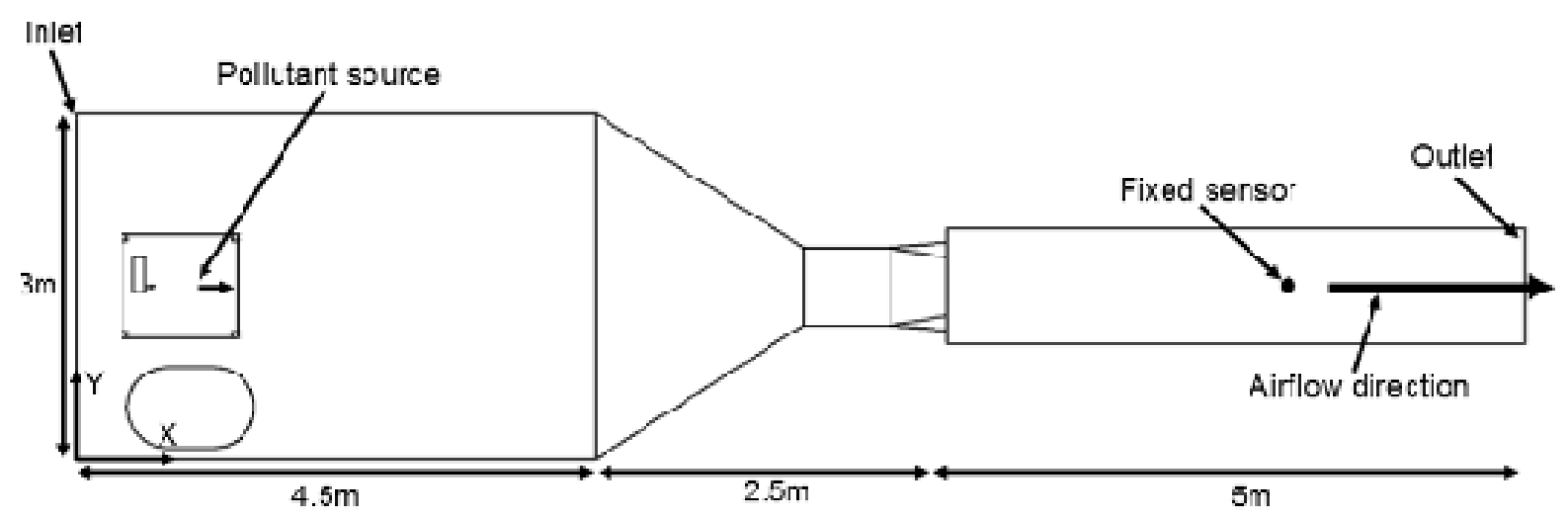

Figure 3 - Schematic drawing of the source injection for CFD application (top view)

\subsection{CFD modeling}

The flow studied in the cabin was incompressible, steady-state and turbulent. It could hence be modeled by an averaged Navier-Stokes approach, associated with the $\mathrm{k}-\varepsilon$ realizable conventional turbulence model [19-20]. The variation in the particle concentration field was modeled by means of the drift-flux model [21] following the transport equation:

$$
\frac{\partial C}{\partial t}(\mathrm{P}, t)+\left[\vec{u}(\mathrm{P})+\tau_{p} \vec{g}\right] \cdot \vec{\nabla} C(\mathrm{P}, t)=\vec{\nabla} \cdot\left(D_{\text {eff }}(\mathrm{P}) \vec{\nabla} C(\mathrm{P}, t)\right)+\frac{Q(t)}{V_{\text {source }}} \chi_{\text {source }}(\mathrm{P})
$$

where $\tau_{p}$ is the response time of the particle, $\vec{g}$ the gravitational acceleration (the $\tau_{p} \vec{g}$ term represents the sedimentation phenomenon). This drift-flux model is similar to equation (1) with only the sedimentation term added. This one depends on position but not on time. The boundary condition 
for wall deposition of particles was modeled using the deposition law of Nerisson et al. [22] which reads:

$$
J=C_{y^{+}} u^{*} V_{d}^{+}
$$

where $J$ is the flux deposited per unit time and per unit area, $C_{y^{+}}$the concentration of particles at the wall, $u^{*}$ the friction velocity, and $V_{d}^{+}$the dimensionless deposition velocity. This one depends on the aerodynamic diameter of the particles, on the surface orientation, on gravity, and on the near-surface velocity but not on concentration (see [23]). Consequently, since equation (28) and its boundary condition (29) are linear with respect to concentration and since their coefficients are time-invariant, the solution of the transport equation has still the form of a convolution product (equation $2 a, b$ ).

For closing the system of equations, Dirichlet conditions are given for the concentration of particles at the air inlets, that is $Y=Y_{\text {background }}$ (corresponding to the uniform initial background concentration in the cabin, that is uniform) at the inlet of the cabin and $Y=Y_{\text {injection }}$ at the injection outlet, which is connected to the injection of the source through the equation:

$$
Y(t)=Q(t) /\left(S_{\text {injection }} u_{\text {injection }}(t)\right)
$$

where $S_{\text {injection }}$ is the outlet section of the divergent nozzle connected to the calibration source and $u_{\text {injection }}$ is the source velocity outlet.

Of course, in order to get a convolutive solution of the form $(2 b)$, the background concentration is substracted from the concentration in the numerical simulation.

\subsection{Generation of the reduced model output}

Two numerical simulations were conducted.

- First, the time-averaged fluid flow was computed: it is then frozen for aerosol computations (the flow is supposed to be unaffected by the presence of particles); The fluid flow is modeled by a RANS, $k-\varepsilon$ realizable turbulence model. 
- Second, two unsteady simulations of the particle flow (recording time step of 0.5 second) were performed. The first was performed to calibrate the impedance, and the second corresponds to the unknown source to be estimated. The drift-flux model (28) with the deposition model (29) are solved to determine the particles flow in the cabin. The particles are monodispersed with a mean diameter of $3.4 \mu \mathrm{m}$ ( $\tau_{p}$ is constant).

Figure 4 summarizes the two particle injection rates that are used in the different numerical simulations (calibration and estimation configurations).

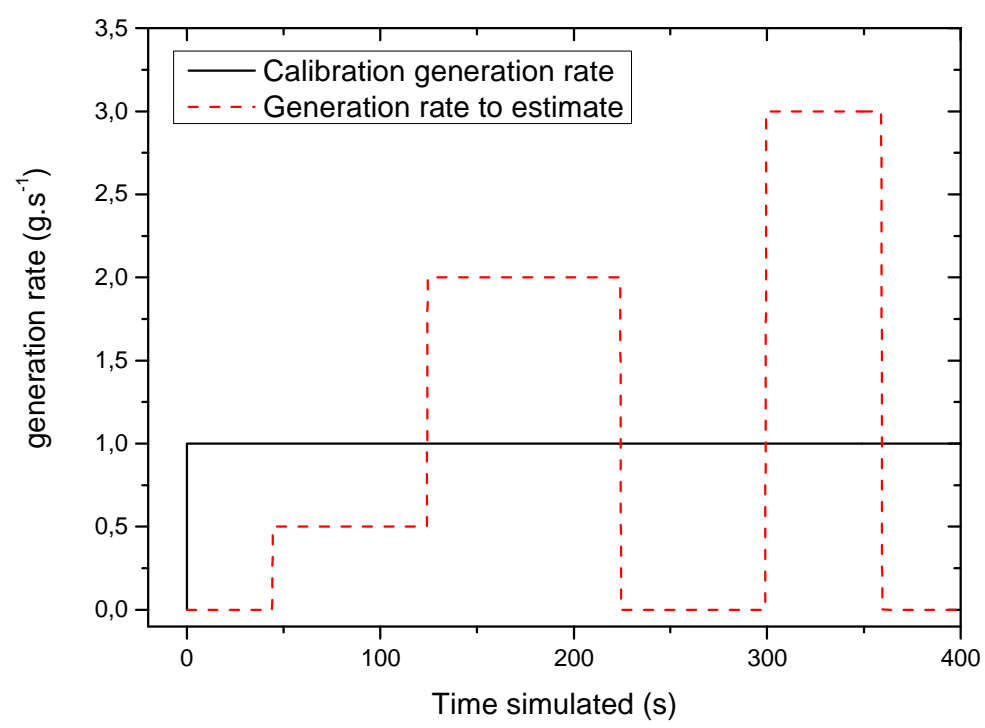

Figure 4 - Mass generation rate of particles used in the numerical simulations for the calibration and the estimation

\subsection{Inversion of synthetic (CFD) concentration signals}

In this section, the method is applied to an ideal CFD case (non-noised concentration). Then, the impact of measurement noise on the calibration and estimation phases is considered.

\subsubsection{Application of the inverse method to non-noised synthetic (CFD) signals}

First step: impedance calibration

Impedance estimation is performed using the injection generation rate $\boldsymbol{Q}^{\text {cal }}$ and the CFD concentration signal $\boldsymbol{Y}^{\text {cal }}$ calculated in the extraction duct (shown in Figure 5). 


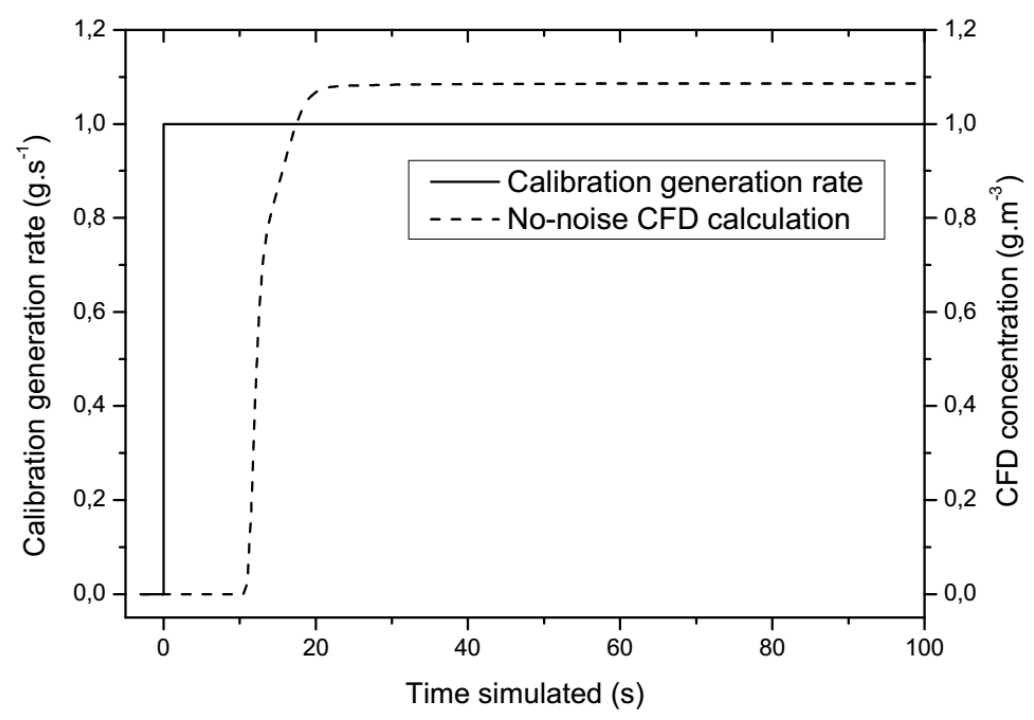

Figure 5 - Signals used for impedance calibration in the ideal case without measurement noise

The impedance calculation (equation 26) is made with a truncation order obtained by Morozov's principle (equation 18c). As no noise is added on the CFD simulation, the standard deviation of the calculated concentration is taken equal to the CFD precision at convergence, that is a value $\sigma=1.010^{-8} \mathrm{~g} \cdot \mathrm{m}^{-3}$. Figure 6 illustrates the determination of the truncation order (with $t_{i}=i \Delta t$ for $i=1$ to $m_{1}$ with $m_{1}=200$ and $\left.\Delta t=0.5 \mathrm{~s}\right)$ using the Morozov's criterion, see equation (18c). The resulting optimal truncation order is $\alpha_{1}=199$ (i.e. one value removed for SVD).

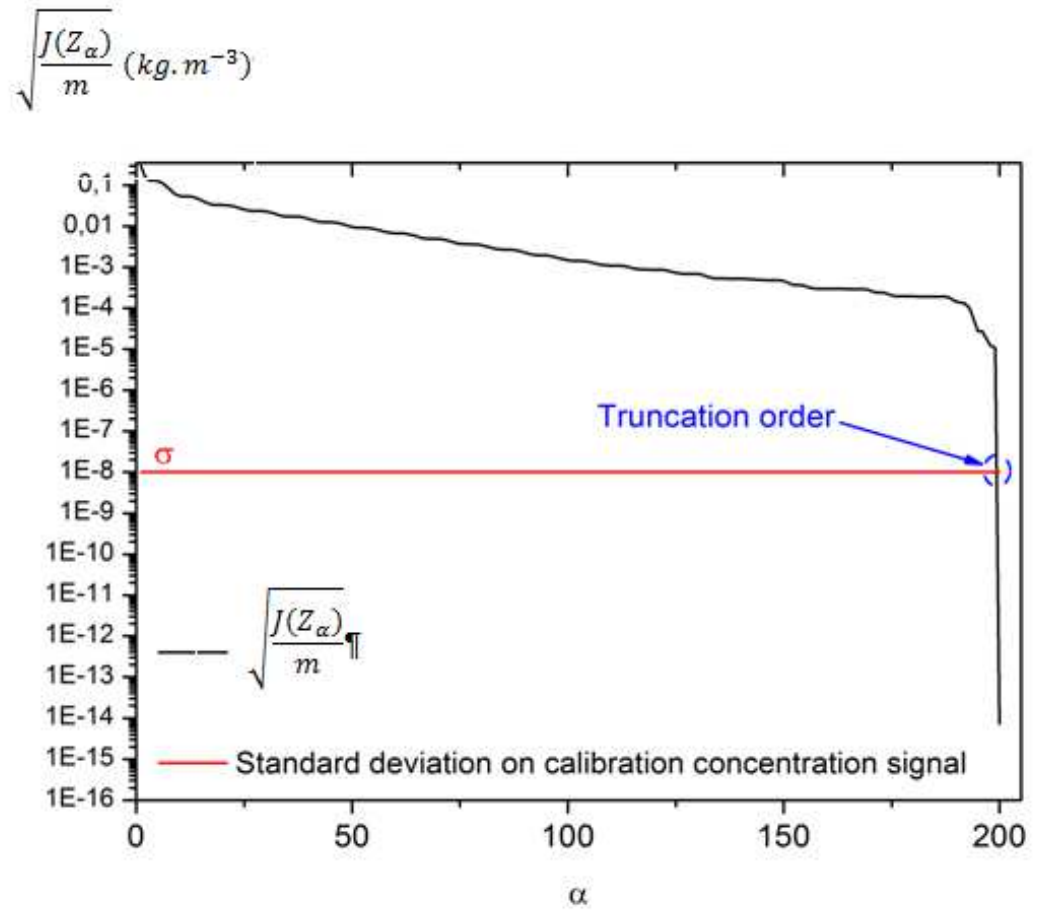


Figure 6 - Truncation order determination for calibration with non-noised signals

Figure 7 shows the corresponding estimated impedance.

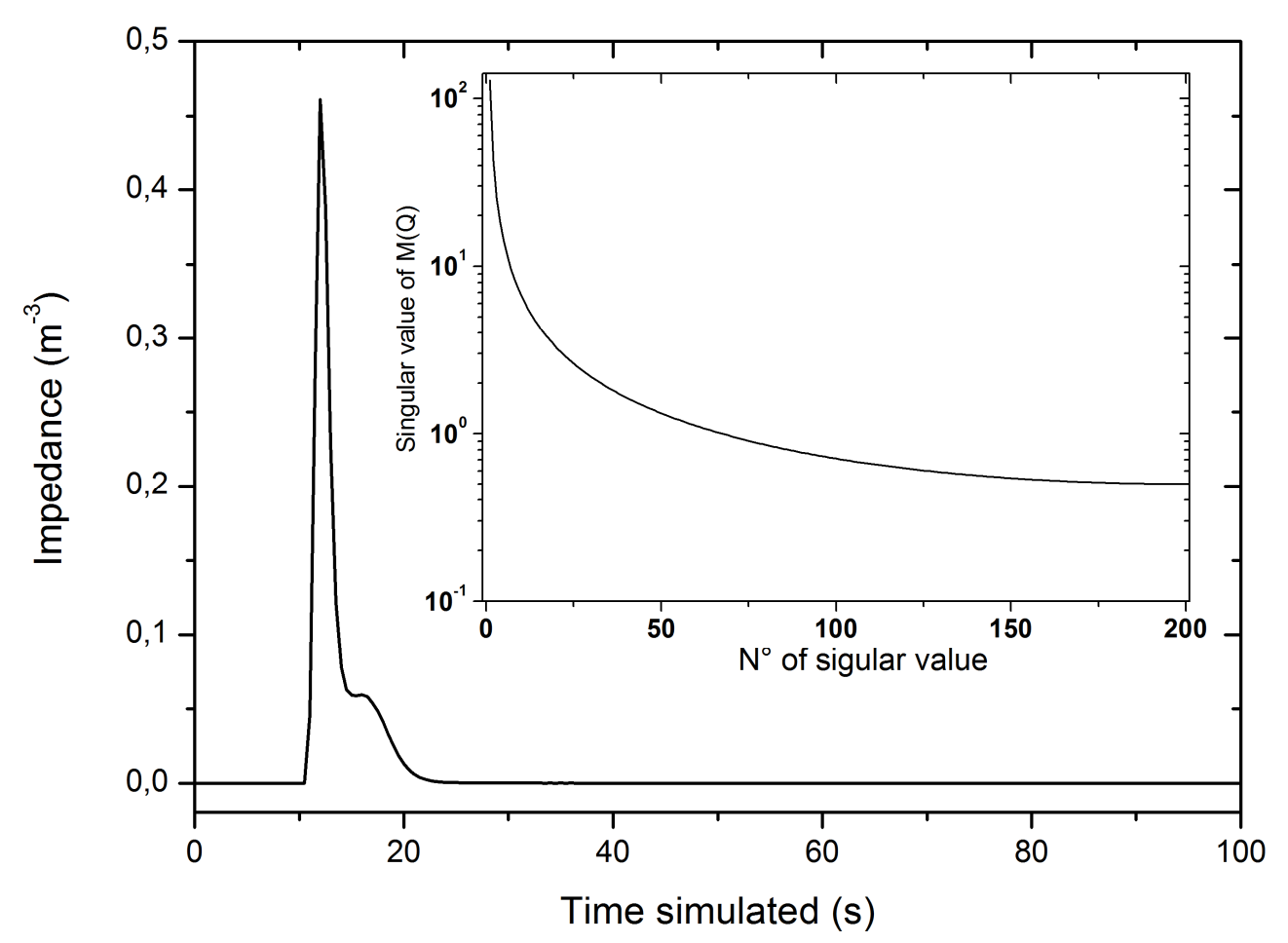

Figure 7 - Ideal impedance $\hat{\boldsymbol{Z}}_{\alpha_{1}}$ estimated from non-noised signals

Let us remind here the significance of an impedance: it is the specific concentration response to a pulse (i.e. a Dirac distribution) of input (an emitted mass flowrate). This can be seen easily by putting $\bar{Q}(p)=1$ in equation (2a), without paying particular attention to the choice of the physical units (the Laplace transform of a Dirac distribution is equal to a constant function in time that is equal to unity).

Here, one can notice a maximum in figure 7 at about a time $t=12 \mathrm{~s}$ after the input start, as well as a second relative maximum (a "bump") at $t=16 \mathrm{~s}$. One can find a physical explanation for both of them: the absolute maximum is nearly equal to the time of flight (that corresponds to a straight line path between source and sensor, see figure 1), while the relative one corresponds to a curved path, in parts of the cabin where velocities are lower (close to the floor or to the ceiling) with a delayed arrival. 
The estimation is carried out from the impedance $\hat{\boldsymbol{Z}}_{\alpha_{1}}$ previously estimated (Figure 7) and from CFD output concentration (see equation (27)) with generation rate shown in Figure 5 as input. To construct the $\mathbf{M}\left(\hat{\boldsymbol{Z}}_{\alpha_{1}}\right)$ matrix, the time step is the same as in the calibration $(\Delta t=0.5 \mathrm{~s})$, with $t_{i}=i \Delta t$ for $i=1$ to $m_{2}$ and with $m_{2}=800$. In this step, the truncation order by Morozov principle is $\alpha_{2}=175$

Thus the zero level of the estimated parameterized impedance has been set for times $t_{i}$ with $m_{1}<i \leq m_{2}$ in the construction of the Toeplitz matrix $\mathbf{M}\left(\hat{\boldsymbol{Z}}_{\alpha_{1}}\right)$, see equation (27). Even if this case is an ideal case for a signal $\boldsymbol{Y}$ without noise, the truncation is higher than in the impedance calibration step $\left(\alpha_{2}<\alpha_{1}\right)$ because of the random error already present in the estimated impedance $\hat{\boldsymbol{Z}}_{\alpha_{1}}$. Figure 8 shows the CFD concentration used and the estimated generation rate.
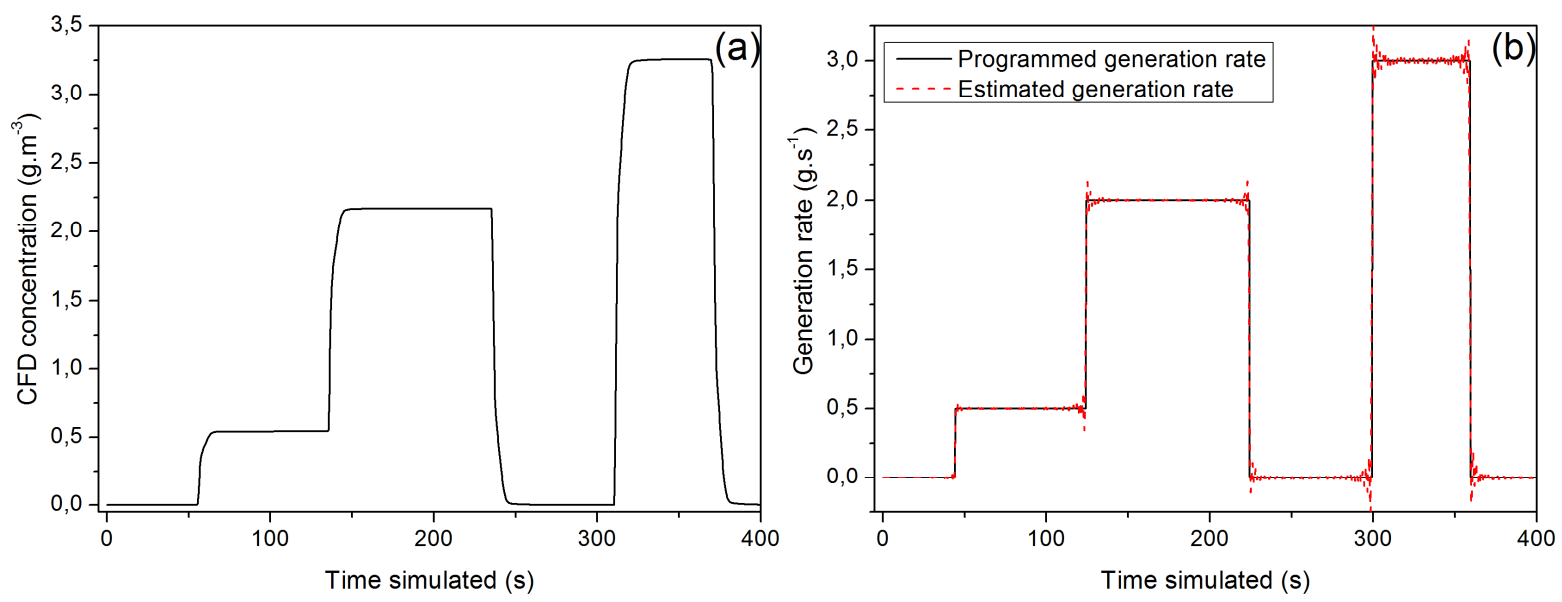

Figure 8 - (a) CFD non-noised concentration used for estimation - (b) Estimated generation rate

As shown in Figure 8, the reconstruction of the injected generation rate is very good. On the estimated curve, small oscillations appear when the generation rate changes suddenly. These are due to noise caused by the solution of the detailed model, which has already affected the truncation order in the calibration phase.

\section{Dose analysis}

The impedance dose calculated by the two methods (equations 22 and 24 in section 3.4 ) is given in Table 1, together with the emission dose estimated in the second step. 


\begin{tabular}{|c|c|c|c|c|c|}
\hline$\hat{D}_{Z}^{a}$ & $\hat{D}_{Z}^{b}$ & & $\begin{array}{c}D_{Q}^{\text {real }} \\
\text { (CFD input) }\end{array}$ & $\hat{D}_{Q}^{a}$ & $\hat{D}_{Q}^{b}$ \\
\cline { 4 - 5 } \cline { 4 - 5 } & \multicolumn{3}{|c|}{$\mathrm{g}$} \\
\hline 1.08 & 1.09 & 420.00 & 420.02 & 418.40 \\
\hline
\end{tabular}

Table 1 - Dose calculation of the impedance and of the unknown emission rate in the ideal numerical case

Both impedance doses are very close (differences below 1\%). The emitted dose given by equation (23) is slightly closer to the true value (CFD input) than the estimation obtained by equation (25). However, the two dose calculation methods provide coherent results with errors lower than 0.4 $\%$. In this case where the concentration signals are noiseless in the calibration and estimation steps, the estimation method of the generation rate gives correct results. The next section concerns the impact of measurement noise on the estimation of the impedance and of the unknown generation rate.

\subsubsection{Application of the inverse method to noised signals}

The signals used are exactly the same as previously with the addition of a noise. Equation (15) defines a measured concentration $\left(Y_{i}^{\text {cal }}\right)$, at a given time for example, as the sum of an exact concentration $\left(C_{i}^{\text {exact }}\right)$ and a noise $\left(\varepsilon_{i}\right)$ with an expected value equal to zero $\left(\mathrm{E}\left(\varepsilon_{i}\right)=0\right)$. The signal-to-noise ratio is defined here as the ratio of Euclidian vector norms as:

$$
\operatorname{SNR}_{2}(Y) \equiv \frac{\left\|\boldsymbol{Y}^{\text {cal }}\right\|_{2}}{\|\boldsymbol{\varepsilon}\|_{2}}=\left(\sum_{i=1}^{m}\left(Y_{i}^{c a l}\right)^{2}\right)^{1 / 2} /\left(\sum_{i=1}^{m} \varepsilon_{i}^{2}\right)^{1 / 2}
$$

Two different signal-to-noise ratios (SNR) are tested here: a very noisy signal with $\operatorname{SNR}_{2}(Y)=5$ and one another less noisy one with $\mathrm{SNR}_{2}(Y)=25$.

First step: impedance calibration

Figure 9 shows the noised concentration for the calibration (a) as well as the estimated impedance (b). An independent identically distributed Gaussian noise of standard deviation $\sigma=\frac{1}{\sqrt{m}}\left\|\boldsymbol{Y}^{\text {cal }}\right\|_{2} / S N R_{2}\left(Y^{c a l}\right)$ was used to produce the added noise vector $\boldsymbol{\varepsilon}$. 

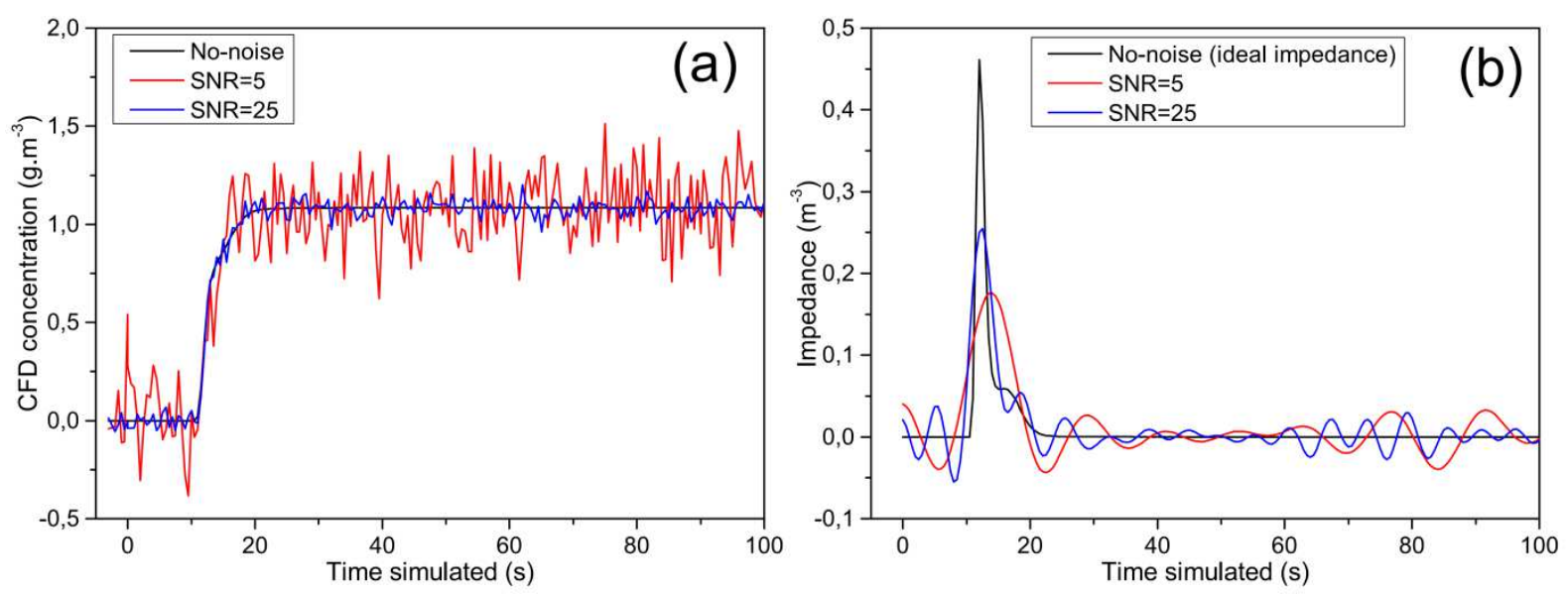

Figure 9 - (a) CFD noised concentration used for calibration - (b) Impedance estimated for different noisy signals with the optimum number $\alpha_{1}$ of singular values

Morozov's principle gives for $\operatorname{SNR}_{2}(Y)=5$ and $\operatorname{SNR}_{2}(Y)=25$ a truncation order $\alpha_{1}$ equal to 15 and 35 respectively (see Table 2). Figure 9b shows that when the SNR is high, the characteristic peak of the impedance is close to the ideal case. Nonphysical oscillations caused by the concentration noise appear before and after the characteristic peak. If the SNR gets very small, the impedance calculation becomes very inaccurate because of an excessive truncation that causes the appearance of non-physical oscillations which blur the characteristic peak. However, if we consider the impedance dose only, the SNR has a very little influence, as can be seen on Table 2.

\begin{tabular}{|c|c|c|c|}
\hline \multirow{2}{*}{$\mathrm{SNR}_{2}(C)$} & $\hat{D}_{Z}^{a}$ & $\hat{D}_{Z}^{b}$ & \begin{tabular}{c} 
Truncation order \\
\cline { 2 - 4 }
\end{tabular} \\
\cline { 2 - 4 } & \multicolumn{2}{|c|}{$\mathrm{s} . \mathrm{m}^{-3}$} & $\begin{array}{c}\alpha_{1} \\
\left(\text { for } m_{1}=200\right)\end{array}$ \\
\hline No-noise & 1.08 & 1.09 & 199 \\
\hline 25 & 1.07 & 1.09 & 35 \\
\hline 5 & 1.12 & 1.09 & 15 \\
\hline
\end{tabular}

Table 2 - Dose calculation of the impedance for different SNR in the CFD case and corresponding truncation order

Even with a very low SNR, both algorithms $\mathbf{a}$ and $\mathbf{b}$ allow to retrieve the impedance dose with $\mathbf{a}$ precision of about $4 \%$. So the main impact of the noise relates to the reconstruction of the impedance but not on the estimation of its dose. 


\section{Second step: unknown source estimation}

Once the impedance has been estimated for different noise levels, the second step of the method is performed on a concentration signal similar to that of Figure 8a but with an added noise corresponding to the tested SNR. Figure 10 shows the noisy concentration signals and the resulting estimated generation rates using the corresponding identified impedances.
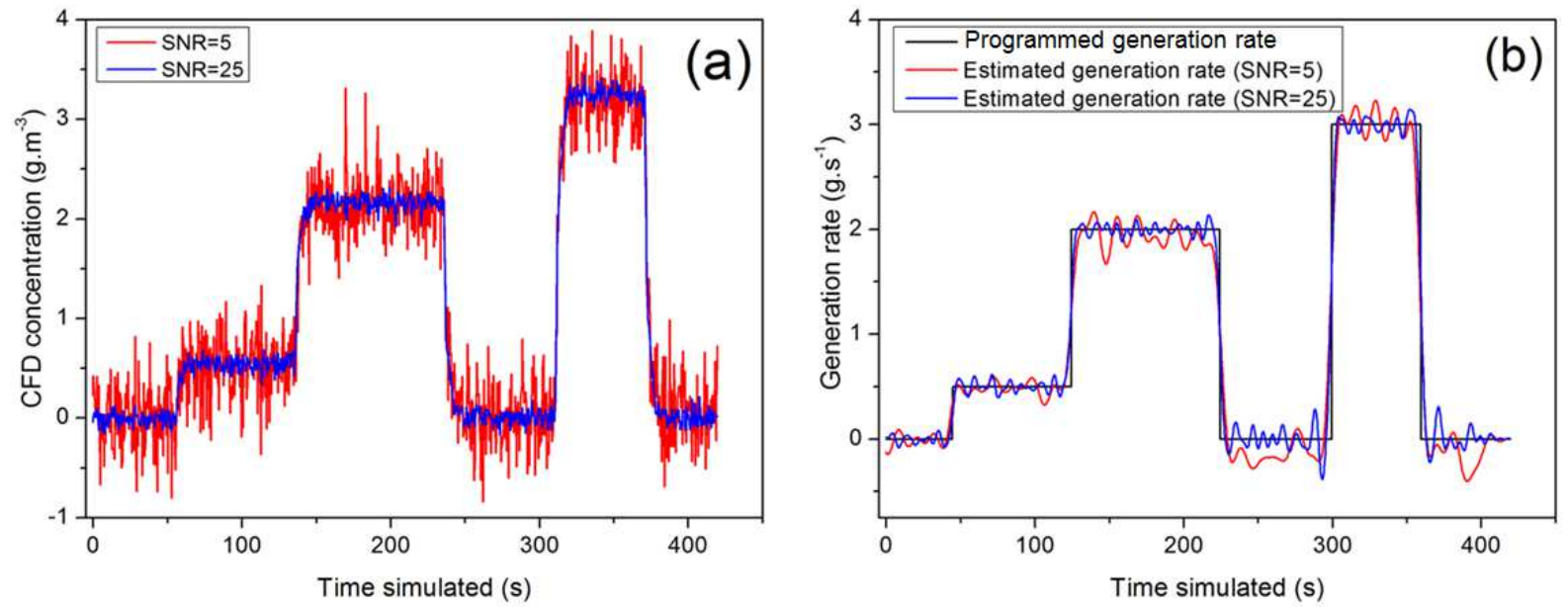

Figure 10 - (a) CFD noised concentration used for estimation - (b) Generation rate estimated for different noisy signals

The optimum truncation orders $\alpha_{2}$ (Morozov principle) are 63 for $\operatorname{SNR}_{2}(Y)=5$ and 91 for $\mathrm{SNR}_{2}(Y)=25$ (see Table 3). So, the conclusion is the same as for the impedance calibration step: the SNR and the optimal truncation orders vary in the same direction. We can observe that the reconstruction of the emission rate seems to be of good quality for each SNR level. However this reconstruction is slightly worse for a low SNR. The effect of SNR on the estimated doses is presented in Table 3.

\begin{tabular}{|c|c|c|c|c|}
\hline \multirow{2}{*}{$\mathrm{SNR}_{2}(C)$} & $\begin{array}{c}D_{Q}^{\text {real }} \\
\text { (CFD input) }\end{array}$ & $\hat{D}_{Q}^{a}$ & $\hat{D}_{Q}^{b}$ & $\begin{array}{c}\text { Truncation order } \\
\alpha_{2} \\
\end{array}$ \\
\cline { 2 - 4 } & \multicolumn{3}{|c|}{$\mathrm{g}$} & \\
\hline No-noise $\left.m_{2}=840\right)$
\end{tabular}

Table 3 - Dose calculation of the unknown generation rate estimated for different SNR's and corresponding truncation orders in the CFD case 
The maximum deviation between the real and the estimated value (for all the presented estimations) is $5.5 \%$. When the measurement noise is low (no-noise or $\operatorname{SNR}_{2}(Y)=25$ ), the estimated dose $\hat{D}_{Q}^{a}$ is more accurate but in the opposite case $\left(\operatorname{SNR}_{2}(Y)=5\right)$ the dose $\hat{D}_{Q}^{b}$ becomes better.

Therefore, the implementated method gives satisfactory results for all noise levels tested. Next section is then dedicated to an application on a real experimental case.

\section{Experimental validation of the method}

After testing the method on an ideal CFD case, we propose to consider its application on a real experimental case. The injection for the two steps (calibration and estimation) is achieved with the aerosol generator Palas RBG-1000 using sanding wood dust. Figure 11 shows the two injection rates that are used in both steps of the method.

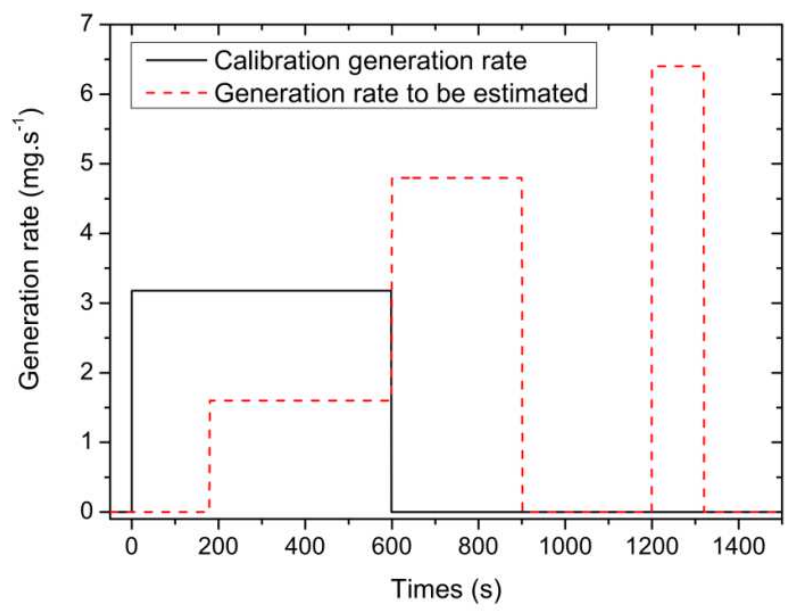

Figure 11 - Mass generation rate of particles in the experimental cases for calibration and source estimation

First step: impedance calibration

In this step, the concentration used is measured by the APS sensor located in the extraction duct. Figure 12a shows the two signals (calibration generation rate and measured concentration) used for impedance calculation. The acquisition time step is $\Delta t=1 \mathrm{~s}$ for $m_{1}=600$ and $t_{i}=i \Delta t$. The concentration signal (Figure 12a) is obtained from an averaged response over 20 tests. This averaging is used to increase the SNR in order to avoid a too ill-posed problem. Let us note that transient particle concentration measurements present indeed very high noise levels that lead to low signal-to-noise ratios. The standard deviation $\sigma=4.16 \mu \mathrm{g} \cdot \mathrm{m}^{-3}$ of the noise has been estimated in a 
statistical way from the fluctuations of the final concentration plateau in this figure. The $\mathrm{SNR}_{2}$ is then equal to 10.44 . So this signal-to-noise ratio lies just in between the two levels tested in the numerical cases considered in section 5. The truncation order obtained by Morozov's principle is $\alpha_{1}=35$.
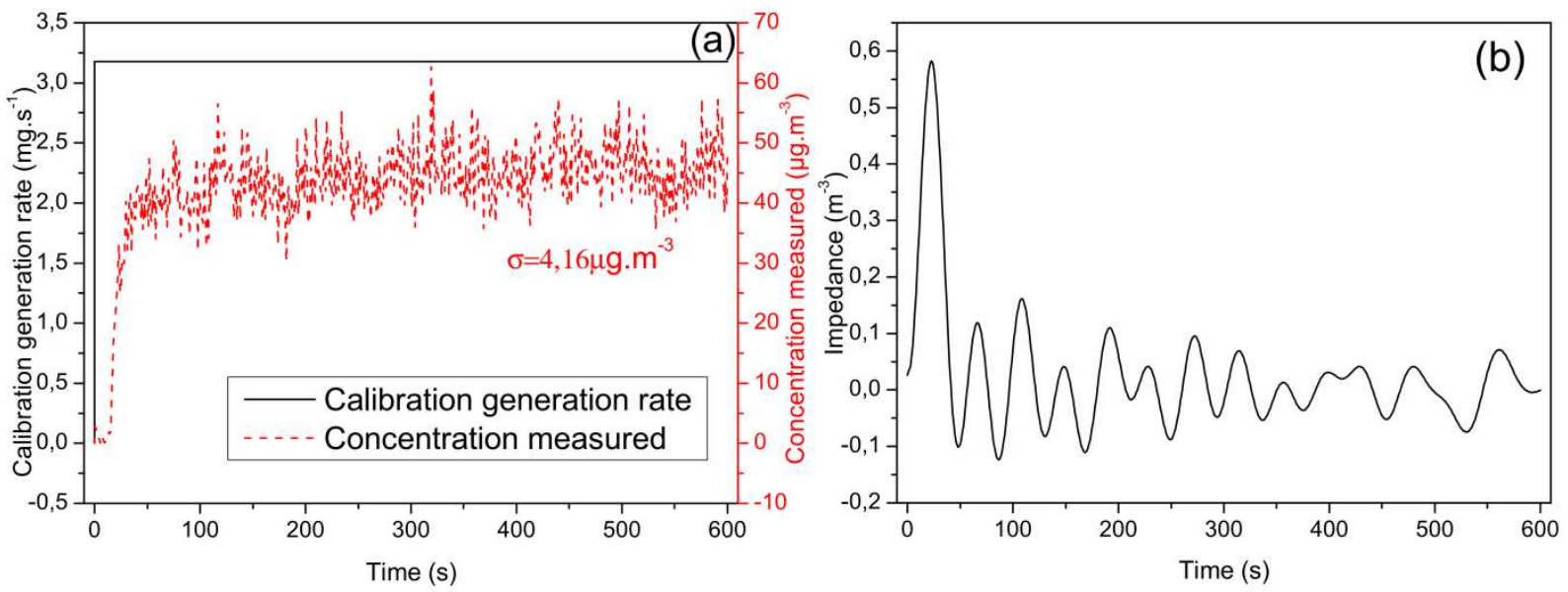

Figure 12 - (a) Signals used for calibration - (b) Estimated impedance

The reconstructed impedance (Figure 12b) exhibits the same type of variation with time as the one obtained previously, with a characteristic peak, but with nonphysical oscillations due to noise in the measurement.

\section{Second step: unknown source estimation}

Figure 13a presents the measured concentration resulting from the unknown injection and Figure $13 \mathrm{~b}$ shows the estimation of the source by the inverse method. The standard deviation $\sigma=3.85 \mu \mathrm{g} \cdot \mathrm{m}^{-3}$ of the concentration noise has been estimated in a statistical way using the first concentration plateau (between $t=215 \mathrm{~s}$ and $t=600 \mathrm{~s}$ ) in the same figure. In this case, $\mathrm{SNR}_{2}=6$ and the resulting truncation order is $\alpha_{2}=55$. 

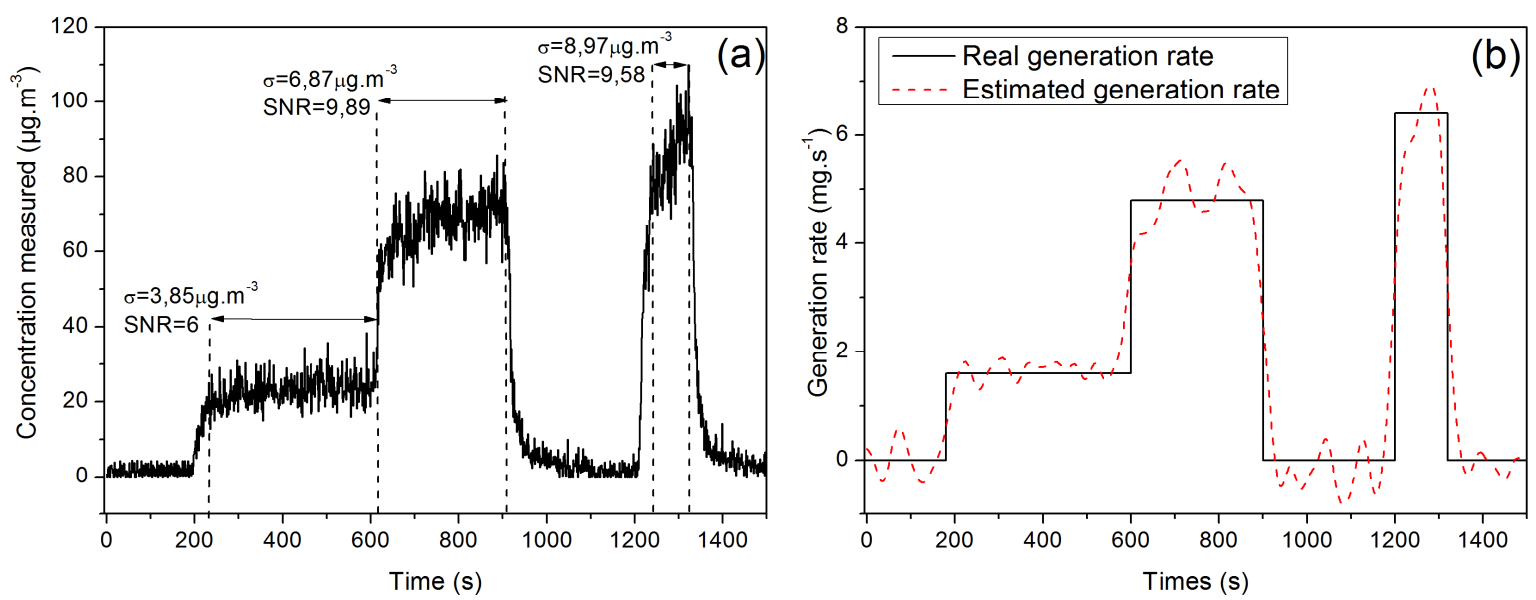

Figure 13 - (a) Experimental concentration used for estimation - (b) Real and estimated generation rate (same particulate source as for the calibration experiment)

The reconstruction of the particle generation rate is in good agreement with the real injection: the three injection plateaus are recovered in the estimation, and the emitted dose is recovered (Table 4).

\begin{tabular}{|c|c|c|c|c|c|c|c|}
\hline$\hat{D}_{Z}^{a}$ & $\hat{D}_{Z}^{b}$ & \multirow{2}{*}{$\begin{array}{c}\text { Truncation } \\
\text { order } \\
\alpha_{1} \\
\text { ( for } m_{1}=600 \text { ) } \\
\text { SNR }=10.44\end{array}$} & & $D_{Q}^{\text {real }}$ & $\hat{D}_{Q}^{a}$ & $\hat{D}_{Q}^{b}$ & \multirow{2}{*}{$\begin{array}{c}\text { Truncation order } \\
\alpha_{2} \\
\text { (for } m_{2}=1500 \text { ) } \\
\text { SNR }=6\end{array}$} \\
\hline \multicolumn{2}{|c|}{$\mathrm{s} . \mathrm{m}^{-3}$} & & & \multicolumn{3}{|c|}{ g } & \\
\hline 14.790 & 15.670 & 35 & & 2.885 & 2.867 & 2.689 & 55 \\
\hline
\end{tabular}

Table 4 - Dose calculation for the impedance and for the unknown source generation rate in the experimental case (same particulate source for calibration and real experiments)

Table 4 shows that the two impedance doses calculated are close. Moreover, the estimated emission doses $\left(\hat{D}_{Q}^{a}\right.$ or $\left.\hat{D}_{Q}^{b}\right)$ are very close to the real emitted mass. The error for $\hat{D}_{Q}^{a}$ and $\hat{D}_{Q}^{b}$ are $0.6 \%$ and $6 \%$ respectively.

So, we see here that use of the transient (a) method gives a better estimate of the emitted dose than the purely steady state (b) method, while the opposite was true in the simulated case shown in Table 2, with a very close $\mathrm{SNR}_{2}$ ratio (5, instead of 6 here). However, no general conclusion about the superiority of one of these methods can be drawn here, since in both cases, only one realization of 2 experimental or simulated estimations (calibration and input estimation) has be made, with high relative noise levels. 
Let us note finally that comparison of Figure 13a (concentration measurement) and $8 \mathrm{a}$ (simulated CFD concentration output) shows that our CFD model was biased because the constant plateaus of its output cannot be found in the experimental measurements.

\section{Conclusions}

The work presented in this paper describes the development of a method for estimating an unsteady injection of particulate pollutant in a ventilated room from transient concentration measurements. This corresponds to a convolution model linking the concentration at any point to the intensity of the source. The developed method is based on two steps:

- The impedance identification, which derives from a controllable source (which the generation rate is known) and a simultaneous concentration measurement in a calibration experiment;

- Estimation of the unknown generation rate, which uses the previously determined impedance and the concentration signal resulting from this unknown generation rate.

For each step, the TSVD regularization of the corresponding inverse linear problem is used. The truncated order is determined by Morozov's discrepancy principle.

First, the method was applied and validated starting from the concentration output of an ideal (nonoise measurement) CFD detailed model where all parameters (ambient settings, injected generation rate, velocity fields ...) are controlled. In this case, a good quality reconstruction of the transient injection rate was reached, with an emitted dose estimated within $0.5 \%$ of the real dose.

Second, the method was used in the CFD case with two different signal to noise ratios $\left(\mathrm{SNR}_{2}\right.$, defined using the Euclidian norm), a high one (25) and a low one (5). For these two cases, the reconstruction of the transient intensity and of the dose could be reached, within $5.5 \%$ for the dose in the most difficult case.

Next, validation of the method was made on an experimental basis, using the same sanding dust injection system in the calibration and unknown source estimation experiments. Even if some assumptions required by the implementation of a convolution model could be questioned, for example a wide distribution $(0.5$ to $20 \mu \mathrm{m})$ of the characteristic size of the non spherical particles with possible 
different diffusion coefficients for each species in the turbulent air flow, the potential of the method was confirmed, with the same qualitative conclusions as above.

In a parallel study, the method has been applied with success on a real sanding experiment, after a calibration experiment with the same sanding dust injection system. These results are not presented here but can be found in [23].

Other versions or other applications of this method can be considered:

- There is no problem to apply the same methodology for a sequential "on line" input estimation: the only change is to replace Truncated Singular Value Decomposition by J. V. Beck's function specification method (also called "Future times" method), which is quite popular for sequential regularized inversion, see [16].

- The deposition rate in the model and in the experiment can be taken into account, with a Robin's boundary conditions at the wall.

- The same type of impedances can be used in order to estimate the time variation of several sources (a number $p$ ) that are active simultaneously in a "real experiment", using at least as many sensor responses (a number $q$ ) as the number of sources (this is commonly called the "source separation problem" in the inverse literature). This requires as many calibration experiments as the number of sources in a first step, with each experiment corresponding to a single active source at the time. In the second step, once the $p \times q$ impedances identified, a superposition of the responses corresponding to each source allows to get a model for the global response of each sensor. Of course, the ill-posed nature of the inverse problem is increased, with respect to each of the individual impedance estimation problem met in the calibration step: two nearby sources will be difficult to separate for example. However, this is possible, as it has been shown experimentally in reference [14] (see the introduction section of this paper). The reduced model used was different (Modal Identification Method), but the underlying physical problem was the same.

- This type of transfer function approach can be applied to other systems based on a transport law (characterization of heat exchangers for example). 


\section{References}

[1] R.-B. Hayes, M. Gerin, J.-W. Raatgever, A. Bruyn, "Wood-related occupations, wood dust exposure, and sino nasal cancer," American Journal of Epidemiology, vol. 124, no 4, p. 569-577, 1986.

[2] C.-H. Barcenas, G.-L. Delclos, R. El-Zein, G. Tortolero-Luna, L.-W. Whitehead, M.-R. Spitz, "Wood dust exposure and the association with lung cancer risk," American Journal of Industrial Medecine, vol. 8, no 1, p. 275-282, 2009.

[3] T. Kauppinen, R. Vincent, T. Liukkonen, and al. "Occupational exposure to inhalable wood dust in the member states of the European Union", Annals of Occupational Hygiene, vol. 50, no 6, p. 549-561, 2006.

[4] A. Thorpeand, R.-C. Brown, "Measurements of the effectiveness of dust extraction systems of hand sanders used on wood", Annals of Occupational Hygiene, vol. 38, 3,. 279-302, 1994.

[5] J.-R. Fontaine, J.-P. Muller, M.-C Braud, P. Brouté, R. Accart, D. Pinsard, G. Marmoret, J.-P. Bayle, P. Bourges, "Hygiène et sécurité du travail," Oct. 2010

[6] B. Besselink, U.Tabak, A.Lutowska, N.vandeWouw, H.Nijmeijer, D.J. Rixen, M.E.Hochstenbach , W.H.A.Schilders, "A comparison of model reduction techniques from structural dynamics", numerical mathematics and systems and control, Journal of Sound and Vibration, 332 (2013) 4403-4422.

[7] J. Atmadjaand A.C. Bagtzoglou, "State of the art report on mathematical methods for groundwater pollution source identification", Environ. Forensics (2001) 2, 205-14.

[8] M. Bocquet, "Reconstruction of an atmospheric tracer source using the principle of maximum entropy", I: Theory, Q.J.R. Meteorol. Soc.(2005) 131, 2191-208.

[9] M. Bocquet, "Reconstruction of an atmospheric tracer source using the principle of maximum entropy", II: Applications, Q.J.R. Meteorol. Soc. (2005) 131, 2209-24.

[10] X.Davoine $X$ and M. Bocquet, "Inverse modelling-based reconstruction of the Chernobyl source term available for longrange transport". Atmos. Chem. Phys. (2007) 7, 1549-64.

[11] E. Lushi, J. M. Stockie, "An inverse Gaussian plume approach for estimating atmospheric pollutant emissions from multiple point sources", Atmospheric Environment 44 (2010) 1097-1107.

[12] A. El Badia, T. Ha-Duong T and A. Hamdi, "Identification of a point source in a linear advection-dispersion-reaction equation: application to a pollution source problem”, Inverse Problems (2005) 21, 1121-36. 
[13] T Maalej, D Maillet and J-R Fontaine, "Estimation of position and intensity of a pollutant source in channel flow using transmittance functions", Inverse Problems (2012) , 28, 055010, doi:10.1088/0266-5611/28/5/055010.

[14] M. Girault, D. Maillet, F. Bonthoux, B. Galland, P. Martin, R. Braconnier and J.-R. Fontaine, "Estimation of time-varying pollutant emission rates in a ventilated enclosure: inversion of a reduced model obtained by experimental application of the modal identification method", Inverse Problems (2008) 24, 015021.

[15] E. Videcoq and D. Petit, "Model reduction for the resolution of multidimensional inverse heat conduction problems", Int. J. Heat Mass Transfer (2001) 44,1899-911.

[16] J.V. Beck, B. Blackwell; C. R., St. Clair Jr., Inverse Heat Conduction. III-Posed Problems. New York, J. Wiley \& Sons, 1985.

[17] R.C. Aster, B. Borchers, C.H. Thurber, Parameter estimation and inverse problems, $2^{\text {nd }}$ edition, Elsevier, 2012.

[18] V.A. Morozov, Methods for Solving Incorrectly Posed Problems, Springer-Verlag, Berlin, 1984.

[19] T.-H.Shih, W. Liou, A. Shabbir, Z. Yang, and Z. Jiang, "A new k-e eddy viscosity model for high Reynolds number turbulent flows", Computers \& Fluids,vol. 24, 227-238, 1995.

[20] J. Zhu and J.-L. Lumley, "A new Reynolds stress algebraic equation model", Computer methods in applied mechanics and engineering, vol. 125, 287-302, 1995.

[21] F. Chen, C.-M Simon, and A.-CK. LAI, "Modeling particle distribution and deposition in indoor environments with a new drift-flux model", Atmospheric Environment, vol. 40, no 2, 357-367, 2006.

[22] P. Nerisson, O. Simonin, L. Ricciardi, A. Douce, and J. Fazileabasse, "Improved CFD transport and boundary conditions models for low-inertia particles", Computers \& Fluids, vol.40, 79-91, 2011.

[23] F. Chata, Estimation of emission profiles of hand held woodworking machines by inverse methods (Estimation par méthodes inverses des profils d'émission des machines à bois électroportatives, in French), PhD thesis, Université de Lorraine, November 27, 2015. 